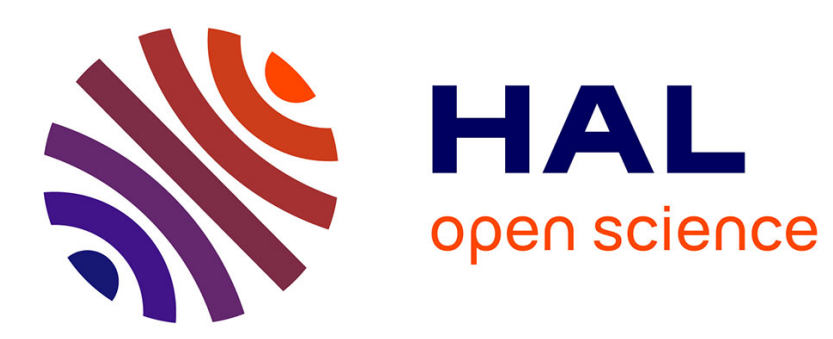

\title{
In-situ measurement of the large strain response of the fibrillar debonding region during the steady peeling of pressure sensitive adhesives
}

Richard Villey, Pierre-Philippe Cortet, Costantino Creton, Matteo Ciccotti

\section{- To cite this version:}

Richard Villey, Pierre-Philippe Cortet, Costantino Creton, Matteo Ciccotti. In-situ measurement of the large strain response of the fibrillar debonding region during the steady peeling of pressure sensitive adhesives. International Journal of Fracture, 2016, 204 (2), pp.175-190. 10.1007/s10704-016-0171-1 . hal-01405061

\section{HAL Id: hal-01405061 \\ https://hal.science/hal-01405061}

Submitted on 1 Dec 2016

HAL is a multi-disciplinary open access archive for the deposit and dissemination of scientific research documents, whether they are published or not. The documents may come from teaching and research institutions in France or abroad, or from public or private research centers.
L'archive ouverte pluridisciplinaire HAL, est destinée au dépôt et à la diffusion de documents scientifiques de niveau recherche, publiés ou non, émanant des établissements d'enseignement et de recherche français ou étrangers, des laboratoires publics ou privés. 


\title{
In-situ measurement of the large strain response of the fibrillar debonding region during the steady peeling of Pressure Sensitive Adhesives
}

\author{
Richard Villey · Pierre-Philippe Cortet · Costantino Creton · Matteo Ciccotti
}

Received: date / Accepted: date

\begin{abstract}
The debonding of Pressure Sensitive Adhesives (PSA) is a classical example of the difficult and unsolved issue of fracture in soft viscoelastic confined materials. The presence of a complex debonding region where the adhesive undergoes cavitation and the very large strain of a spontaneously formed fibrillar network has defied many modeling attempts over the past 70 years. We present here a novel technique to provide an accurate measurement of the local large strain response of the fibrillar debonding region during the steady-state peeling of a well known commercial adhesive over a wide range of peeling velocity and angle. The technique is based on high resolution imaging of the debonding region during peeling and is coupled to a cohesive zone modeling of the adhesive interaction between the flexible tape backing and the rigid substrate. The resulting database provides a strong ground for validating and further developing models (Villey et al. 2015) aiming to capture the effects of both geometry and non-linear adhesive rheology on the exceptional adherence energy of PSAs.
\end{abstract}

Keywords Pressure sensitive adhesive - Large strain · Peeling · Cohesive Zone · Adherence energy

Online Resources available: OR1: movie of a peeling experiment with model superimposed; OR2 mathematical demonstrations.

R. Villey $\cdot$ C. Creton $\cdot$ M. Ciccotti

Laboratoire SIMM, CNRS, ESPCI Paris, PSL Research University, 10 rue Vauquelin, Paris, France

Laboratoire SIMM, Université Pierre et Marie Curie, Sorbonne-

Universités, 10 rue Vauquelin, Paris, France

E-mail: matteo.ciccotti@espci.fr

R. Villey · P.-P. Cortet

Laboratoire FAST, CNRS, Univ. Paris-Sud, Université Paris-Saclay

91405 Orsay Cedex, France

\section{Introduction}

Peeling is a widespread testing method to characterize the adherence properties of all sorts of thin bonded films, certainly due to its easiness of implementation and quantitative evaluation (Kendall 1975; ISO 8510-1; ISO 8510-2; Creton and Ciccotti 2016). However, the weak transposability of peeling test results to predict the adherence performance of a given adhesive joint under different loading geometries such as shear lap, mode I cleavage between rigid beams or probe tack is an important concern for applications. The difficulties of providing a clear and robust mechanical model and interpretation of the peeling strength of a given material layer or joint have focused many efforts since the 50's and no clear picture is available to cover all applications in a comprehensive way (Kaelble 1959; Kaelble 1960; Kaelble 1965; Yarusso 1999; Barquins and Ciccotti 1997; Amouroux et al. 2001; Villey et al. 2015; Derail et al. 1997; Derail et al. 1998; Gent and Hamed 1977; Gent and Petrich 1969).

Despite the apparent simplicity of the peeling geometry at the macroscopic scale and the unambiguous measurement of the injected external work during the test, the structure, applied deformation and stress fields of the debonding region are quite complex and dramatically change when considering different materials. Moreover, when considering the peeling of soft films such as most adhesive tapes - which are the object of the present investigation - these are invariably backed by a thin layer made of a stiffer material in order to limit stretch at a macroscopical scale. The adhesive material in the debonding region is thus subject to a progressive loss of confinement and consequently to a variable degree of stress triaxiality as it is debonded. These position dependent stress and strain fields are coupled to the local bending profile of the backing and are related in a very sensitive way to the macroscopic angle of application of the peeling force, leading to variable damage scenarios. For ex- 


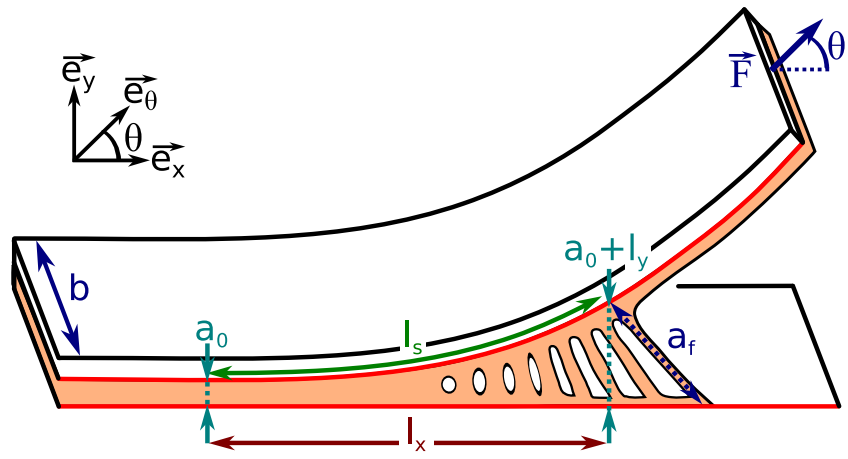

Fig. 1 Model geometry of a peeling experiment. The extremity of the peeled tape is pulled at a velocity $V \overrightarrow{e_{\theta}}$ tilted of an angle $\theta$ with respect to the substrate (along $\overrightarrow{e_{x}}$ ), which is itself translated at velocity $V \overrightarrow{e_{x}}$. $\vec{F}$ is the peeling force at the pulling extremity of the tape. During peeling, the adhesive layer is stretched over a region called the "debonding region", of curvilinear length $l_{s}$ along the tape backing. The adhesive material, of initial thickness $a_{0}$, is strained and forms fibrils up to a maximum length $a_{f}$ at the debonding front. We note $l_{y}$ the elevation of the tape backing at the end of the debonding region and $l_{x}$ the projection of $l_{s}$ on the substrate.

emple, the adhesive debonding region during the peeling of common office tapes undergoes a complex process of cavitation and fibrillation at the microscopic scale such as illustrated in Fig. 1, which leads to a still poorly understood transition between the very stiff oedometric response preceding cavitation and the almost uniaxial response in the unconfined fibrillar region (Villey et al. 2015). The formation of the complex fibrillar structure is very similar to that observed in probe tack experiments, which present stresselongation curves characterized to the first order by a strainrate dependent stress plateau up to a limited elongation before debonding (Creton and Ciccotti 2016). While comparative studies between the uniaxial elongational behavior and probe tack experiments have been carried out for several types of PSA (Lindner et al. 2006; Deplace et al. 2009; Chiche et al. 2005), the development of such a fibrillar pattern during the propagation of a peeling front is more complex since it is strongly coupled with the local bending of the backing, implying variable degree of confinement and level of local stress (Villey et al. 2015).

The aim of the present work is to develop new tools to finely characterize the damage mechanisms at the scale of the debonding region during steady-state peeling of PSA, combining real-time microscopic imaging of the debonding region and a sound modeling of the mechanical interaction between the bending of the elastic backing and the deformation of the fibrillar network, which will be treated as an effective non-linear cohesive zone. A careful compromise between the image resolution on such a small region and the relevant mechanical information coming from the present knowledge on the structure and behavior of the fibril network will be the guide to the identification of a minimal yet rich set of local parameters of the debonding region, namely the local radius of curvature of the backing, the effective average stress applied by the fibrils on the backing, the average strain rate of the fibrils, their maximum extension and tilt angle before debonding.

In this work we investigate the variations of these local parameters via a series of tests on a well known commercial tape over a large range of peeling angle and velocity, in order to discuss the soundness of the estimated parameters in light of the known rheological behavior of such an adhesive and of visual comparisons with the imaging of the fibrils. The robustness and reproducibility of the estimated parameters represent a real experimental breakthrough and become a key to the sound modeling of the adherence properties of peeled tapes as a function of the large strain rheology of the adhesive material and of the surface properties of the adherends (Villey et al. 2015). These enriched parameters can also be used to understand the relationships between the peeling and the tack properties of each adhesive (Creton and Ciccotti 2016).

\section{Microscope filmed peeling experiments}

Peeling experiments are performed at room temperature $\left(23 \pm 2{ }^{\circ} \mathrm{C}\right)$ on a commercial PSA $\left(3 \mathrm{M} \mathrm{Scotch}{ }^{\circledR} 600\right)^{1}$ frequently studied in the literature (Barquins and Ciccotti 1997; Amouroux et al. 2001; Cortet et al. 2007; Cortet et al. 2013; Dalbe et al. 2014a; Dalbe et al. 2014b; Dalbe et al. 2015; Villey et al. 2015). The adhesive tape is peeled from a flat bar by an Instron testing machine (model 3343), which records the peeling force $F$ while imposing a constant pull-out velocity $V \in[3: 3000] \mu \mathrm{m} \mathrm{s}^{-1}$. The bar, tilted of an angle $\theta \in\left[30^{\circ}: 150^{\circ}\right]$ with respect to the pull axis of the testing machine, is mounted on a translation stage: it is translated at the same velocity $V$, resulting in a steady peeling at constant angle $\theta$ (see Fig. 1).

These experiments give access to the peeling force $F(\theta, V)$ from which we compute the energy release rate $G$ associated to the peeling of a unit surface

$G=\frac{F}{b}(1-\cos \theta)$,

where $b=19 \mathrm{~mm}$ is the tape width (see Fig. 1). This expression of $G$ accounts for the work of the operator, but discards the contribution associated to the elastic energy stored in the peeled tape backing elongation (Kendall 1975), which is never larger than $1 \%$ of $G$ in the reported experiments.

Experiments are performed in a range of velocity $V$ where peeling is steady, i.e. where no stick-slip dynamical instability is observed (stick-slip appears at larger velocities for

\footnotetext{
1 This PSA is made of an acrylic adhesive layer of thickness $a_{0} \simeq$ $20 \mu \mathrm{m}$ coated on a UPVC backing of comparable thickness, Young's modulus $E \simeq 2.9 \mathrm{GPa}$ and width $b=19 \mathrm{~mm}$.
} 
this PSA (Dalbe et al. 2014b)). In these conditions the energy release rate $G$ defines an adherence energy $\Gamma(\theta, V)$ that can also be considered as an effective fracture energy for the peeling of the whole adhesive joint (backing, adhesive and substrate) at velocity $V$ and angle $\theta$

$G=\Gamma(\theta, V)$.

The substrate of the adhesive tape to be peeled is made of a first layer of the same PSA tape, which has been applied on the peeling bar following the protocol described in (Villey et al. 2015). The substrate tape layer is changed after each peeling experiment in order to preserve the integrity of its release coating. This procedure enables comparison with previous measurements made by peeling directly from the commercial roller (Barquins and Ciccotti 1997; Amouroux et al. 2001; Cortet et al. 2007; Cortet et al. 2013; Dalbe et al. 2014a). It also ensures a moderate level of adhesion $\left(F \in[0.3: 4] \mathrm{N}, \Gamma \in[20: 80] \mathrm{J} / \mathrm{m}^{2}\right)$, which results in interfacial adhesive failure only, with no residuals on the substrate, even at peeling velocities $V$ as low as few $\mu \mathrm{m} \mathrm{s}^{-1}$.

The shape of the tape backing profile close to the debonding region (where the adhesive is deformed before debonding) is monitored during the peeling experiments using a $1624 \times 1228$ pixels $^{2}$ camera equipped with a microscope objective (frame rate between 5 and $30 \mathrm{fps}$, resolution between 0.55 and $2.2 \mathrm{px} / \mu \mathrm{m})$. For each frame of the movies (such as those represented in Figs. 2 and 3), the outer profile of the tape backing is measured using a binarization algorithm that detects the interface between the dark background and the illuminated tape. The tape backing profile does not significantly change with time during steady peeling (see the movie in Online Resource 1). We can thus average this profile over the entire movie (several hundreds of frames), which removes detection imperfections (related e.g. to the presence of micrometric dusts) and eventually results in a significant increase of the signal-to-noise ratio. This average profile is reported in yellow in Figs. 2 and 3.

In these two figures, we also report the local angle $\alpha$ made by the averaged profile with the substrate as a function of the curvilinear abscissa $s$ along the tape backing in the reference frame of the laboratory (see Fig. 4), related by

$d s \vec{t}=d s \cos (\alpha) \overrightarrow{e_{x}}+d s \sin (\alpha) \overrightarrow{e_{y}}=d x \overrightarrow{e_{x}}+d y \overrightarrow{e_{y}}$.

\section{Simple elastica model}

We first compare the experimental profiles to the theoretical expression of an inextensible elastica beam (Love 1944) with a simple clamping boundary condition at $s=s_{0}$

$\alpha(s)=\theta-4 \arctan \left[\tan \left(\frac{\theta}{4}\right) \exp \left(-\frac{s-s_{0}}{r}\right)\right]$, with

$r=\sqrt{\frac{E I}{F}}$

the buckling length of the tape backing of bending modulus $E I$ submitted to a force $F$, which is the typical value of the curvature radius of the backing close to the debonding region. We actually fit the $\alpha(s)$ experimental profiles outside of the debonding region (sufficiently far from the last visible fibrils) using $s_{0}$ and $r$ as fitting parameters. The result is reported in green in Figs. 2 and 3 where the elastica profiles are extrapolated down to the substrate. The matching between experimental and elastica profiles outside of the debonding region is excellent (see Fig. 2 (f)) and, since we measure the peeling force $F$ independently, the fit of the buckling length $r$ provides a measurement of the bending modulus $E I$ of the tape backing for the portion of the tape being peeled ${ }^{2}$.

As it can be observed in Figs. 2 and 3, the theoretical profiles of the simple elastica model of Eq. 4 clearly do not match the experimental ones in the debonding region. In particular, the profile of the simple elastica model presents a curvature discontinuity at the junction with the substrate where $s=s_{0}$, with a maximum curvature at $s=s_{0}^{+}$. On the contrary, the experimental profiles present no curvature discontinuity and the maximum curvature is remote from the beginning of the debonding region (yet still inside it). It is important to note that, in Figs. 2(f) and 3(h), the origin of the curvilinear abscissa $s$ of the experimental profiles has been set by fitting the profile of the backing by an elastica model with a cohesive zone, as described in section 4 , and not by simple visual observation of the fibrillated region from the side.

The reason for the observed discrepancy with the simple elastica model is obviously related to the existence of forces applied on the tape backing by the stretched adhesive material in the debonding region, which have not been taken into account. Note that the elastic extension of the tape backing (neglected in the simple elastica model) cannot account for the above-mentioned discrepancies, since the tape extension is very small in our experiments. This can be verified by comparing the tape extension $\varepsilon d x=d L$ of a portion $d x$

2 We find that this modulus changes significantly between different rollers, and even between distant portions of the same roller, with an average value $E I=(3.7 \pm 1.7) 10^{-8} \mathrm{~N} \mathrm{~m}^{2}$. This value agrees with the estimate that can be made from the expression $E I=E b h^{3} / 12$, where $E$ is the Young's modulus and $h$ the thickness of the tape backing, using an independent tensile test giving $E b h=1100 \pm 200 \mathrm{~N}$ and microscopic observations of the thickness providing $h=20 \pm 5 \mu \mathrm{m}$. An attentive study reveals that the variations in bending modulus $E I$ can actually be attributed to small variations of the tape backing thickness of amplitude $\pm 3 \mu \mathrm{m}$, since $E I \propto h^{3}$. In any case, in the present study the bending modulus $E I$ will enter the problem only through the parameter $r=\sqrt{E I / F}$, which will be directly measured for each experiment through elastica fits of the peeled tape outside of the debonding region. 

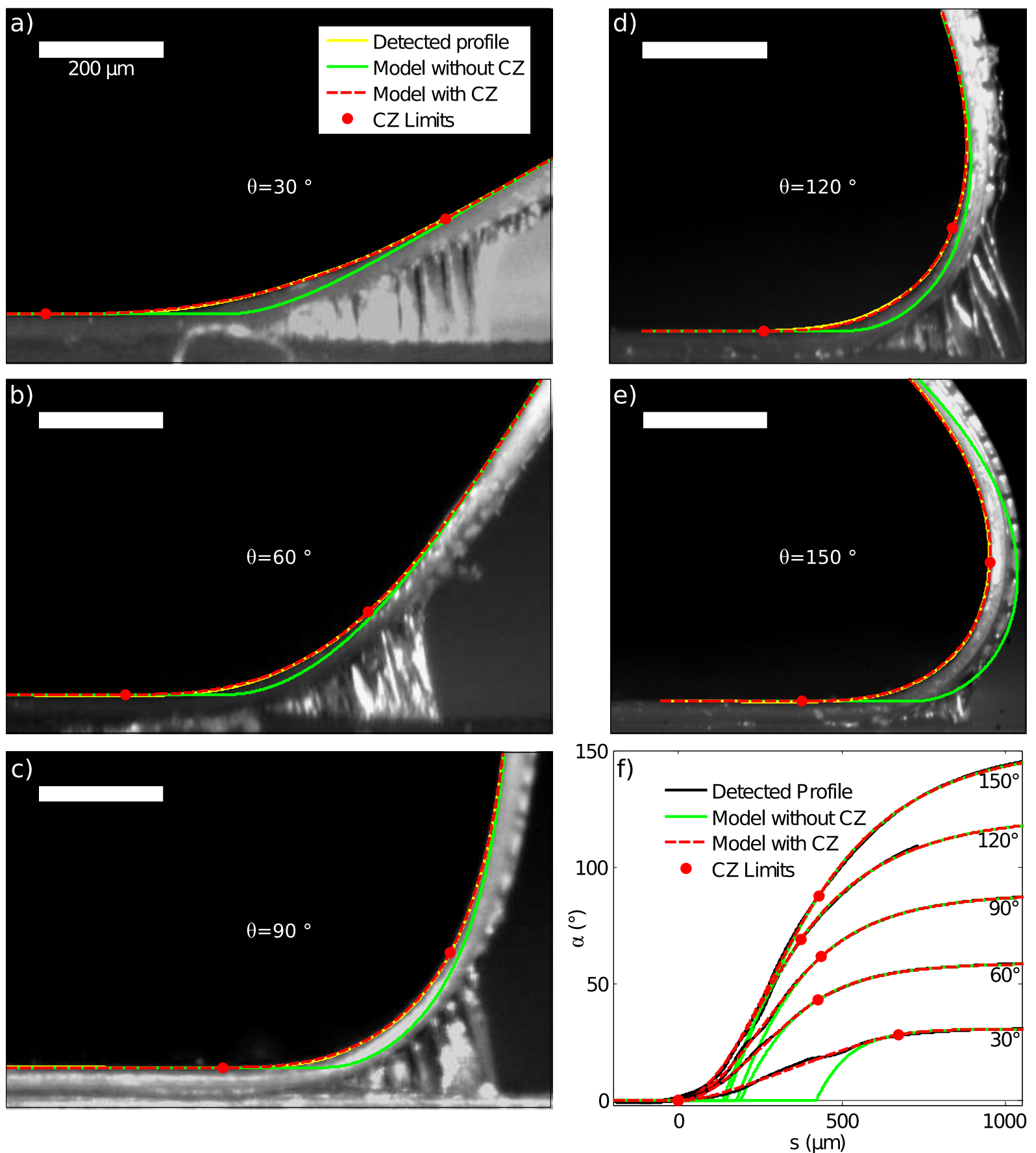

Fig. 2 (a)-(e): Pictures of the debonding region during peeling experiments at $V=100 \mu \mathrm{m} \mathrm{s}^{-1}$ and different peeling angles $\theta$. The profile of the tape backing (reported in yellow) is obtained from an average over several hundreds of detected profiles (one for each frame of the movie). This profile is compared to the classical elastica model of Eq. 4, represented in green. We also report the best fit of the profile using the model of elastica with a cohesive zone presented in Sec. 4 (red dashed lines). This model has two fit parameters, namely the limits of the cohesive zone (CZ), which are highlighted by red circles. In (f), we represent the same data as in (a)-(e) in curvilinear coordinates (see Eq. 3), showing the slope $\alpha$ of the tape backing as a function of curvilinear abscissa $s . s=0$ is set at the beginning of the fitted cohesive zones. 

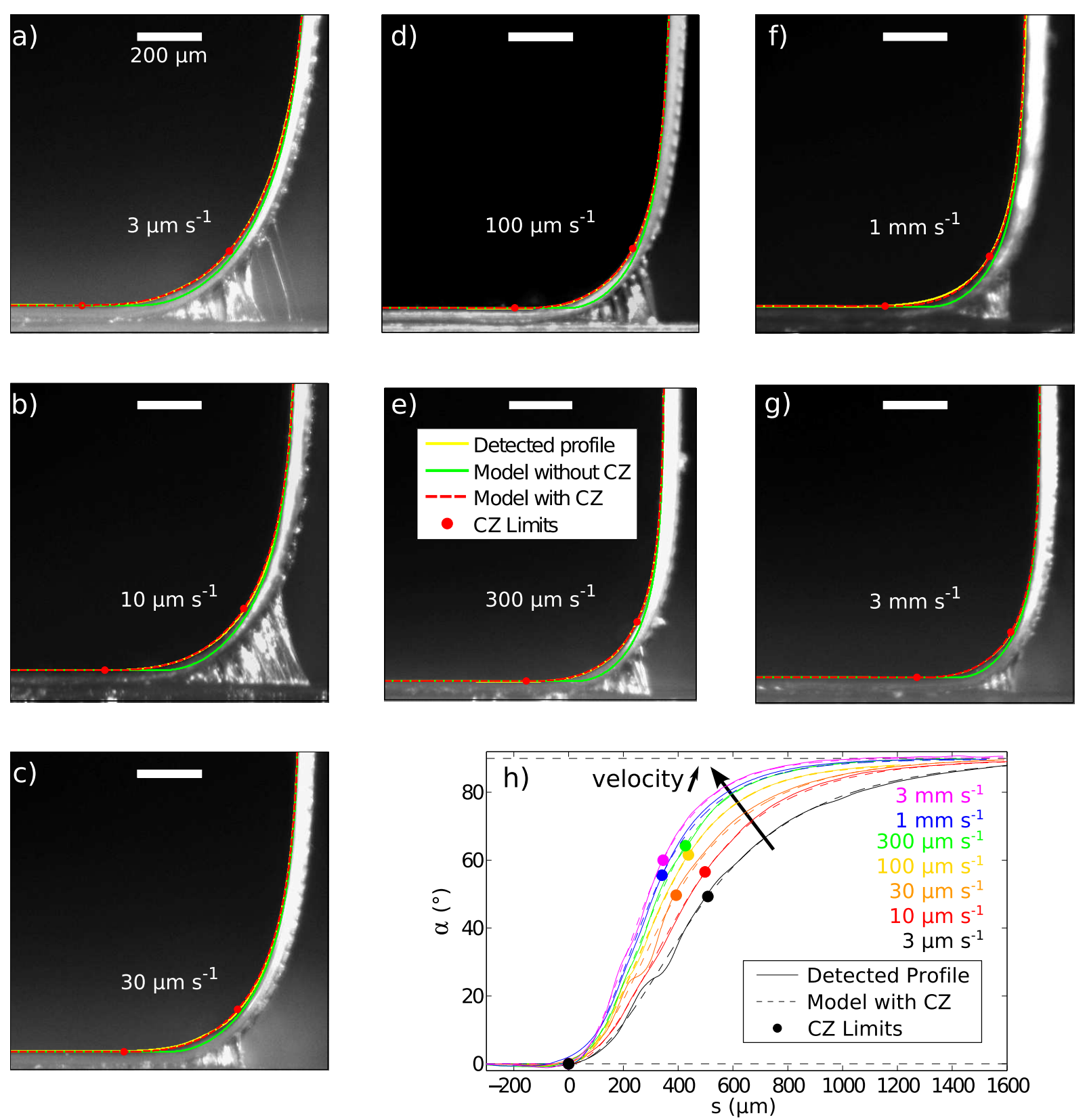

Fig. 3 (a)-(g): Pictures of the debonding region during peeling experiments at $\theta=90^{\circ}$ and different peeling velocities. The color and symbol code is the same as in Fig. 2. In (h), we show the profiles from (a)-(g) in curvilinear coordinates (see Eq. 3 ), with $s=0$ taken at the beginning of the fitted cohesive zones.

of the tape to its vertical deflection $d y$ due to bending: with $F \leq 4 \mathrm{~N}$ and $E b h \simeq 1100 \mathrm{~N}$, the tape relative extension $\varepsilon$ is always smaller than $0.4 \%$ (in the worst case), which can be safely neglected compared to the tape deflection due to bending $\tan \alpha=d y / d x$ as soon as $\alpha$ is larger than $1^{\circ}$.

\section{A model of elastica with a cohesive zone}

In this section we account for the stress distribution $\sigma(s)$ applied to the tape backing in the debonding region in terms of a cohesive zone coupled to the inextensible elastica model.
As a first approximation we consider a uniform effective stress $\bar{\sigma}$ in the debonding region, which corresponds to a traction-separation curve that is constant up to a maximum elongation at debonding, where it drops to zero. The relevance of such an approximation relies on the fact the adhesive material experiences a relative stretching of several hundred percents in the majority of the debonding region (see Figs. 2 and 3), which for confined soft incompressible materials is always associated with fibrillation. This loading condition is very similar to the probe-tack test, which for typical PSA provides stress-strain curves dominated by 


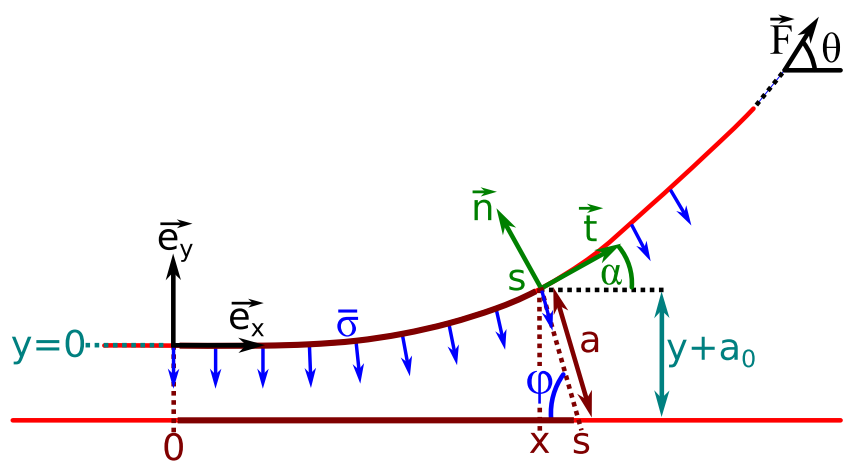

Fig. 4 Cohesive zone model of the debonding region expressed in the reference frame of the laboratory, where the peeling profile is steady. $s=x=0$ is set at the beginning of the cohesive zone. The tape is undeformed at $x \leq 0$ and submitted to a force $\vec{F}$ far from this undeformed zone. The adhesive response is modelled by a uniform stress $\bar{\sigma}$ for $0 \leq s \leq l_{s}$. We assume this stress to follow the fibrils orientation, given by the angle $\varphi$.

a large plateau for the stress (Lakrout et al. 1999; Creton and Ciccotti 2016). One should note that, because of cavitation and fibrillation, $\bar{\sigma}$ does not represent a true local stress, but the average force per unit area acting on the boundaries of the adhesive material (both for peeling and probe-tack experiments).

Since the adhesive presents a fibrillar structure in the majority of the debonding region, it is reasonable to represent the stress applied to the tape backing as a vector that is locally oriented along the fibrils (and with constant modulus $\bar{\sigma})$. Moreover, since the fibrils do not appear to slide on the tape backing nor on the substrate (before debonding), a fibril attached on the tape backing at curvilinear abscissa $s$ should also be attached to the substrate at position $x=s .^{3}$ This enables to compute the fibrils length $a(s)=\sqrt{(s-x(s))^{2}+\left(a_{0}+y(s)\right)^{2}}$ and the angle $\varphi(s)=\arctan \left(\frac{a_{0}+y(s)}{s-x(s)}\right)$ that the fibrils form with the substrate (see Fig. 4). Note that $x(s)=\int_{0}^{s} \cos (\alpha(t)) d t$ and $y(s)=\int_{0}^{s} \sin (\alpha(t)) d t$ are the horizontal and vertical coordinates of the point along the tape at curvilinear abscissa $s$. In the initial part of the debonding region (before fibrillation) the forces applied by the adhesive are essentially vertical and $\varphi$ remains a valid estimate of the stress orientation, even if fibrils are not present, since the tape backing is only slightly bent so that $s \simeq x$ and $\varphi \simeq 90^{\circ}$.

\footnotetext{
3 The validity of this assumption is limited to the inextensible tape approximation of our model, that follows the arguments provided in section 3 .
}

Our model can then be described by the following system

$$
\begin{aligned}
& E I \alpha^{\prime \prime} \overrightarrow{e_{z}}+\vec{t} \wedge \vec{R}=\overrightarrow{0}, \\
& \vec{R}^{\prime}\left(0<s<l_{s}\right)=\bar{\sigma} b\left[-\cos (\varphi) \overrightarrow{e_{x}}+\sin (\varphi) \overrightarrow{e_{y}}\right], \\
& \vec{R}\left(s \geq l_{s}\right)=\vec{F}, \\
& \alpha(s<0)=0,
\end{aligned}
$$

where' is the derivative with respect to $s$ and $\vec{R}(s)$ is the internal force, applied by the half-part $\tilde{s}>s$ of the tape on the other half-part $\tilde{s}<s$. The first line of this system is the classical beam flexure equation (Love 1944). The second line accounts for the presence of the uniform external stress $\bar{\sigma}$ in the cohesive zone between $s=0$ and $s=l_{s}$ (in curvilinear abscissa). The two remaining lines represent the boundary conditions. A combination of the first three equations of (6) gives the master equation of our model

$E I \alpha^{\prime \prime}+F \sin (\theta-\alpha)-\bar{\sigma} b \int_{s}^{l_{s}} \sin [\alpha(s)+\varphi(t)] d t=0$.

Eq. 7 is valid in the cohesive zone $s \in\left[0 ; l_{s}\right]$ and should be associated with the description of the rest of the tape backing by

$$
\begin{aligned}
\alpha & =0, & & \text { for } s \leq 0, \\
E I \alpha^{\prime \prime}+F \sin (\theta-\alpha) & =0, & & \text { for } s \geq l_{s},
\end{aligned}
$$

where the first line represents the absence of flexure in the bonded part of the backing out of the debonding region, while the last line is the simple elastica equation in the freestanding part of the backing.

Integration of the second line of (8) gives

$\alpha\left(s \geq l_{s}\right)=\theta-4 \arctan \left[X \exp \left(-\frac{s-l_{s}}{r}\right)\right]$,

where the constant $X$ has to be determined from the continuity relations at the end of the cohesive zone $s=l_{s}$

$$
\begin{aligned}
\alpha\left(l_{s}^{-}\right) & =\alpha\left(l_{s}^{+}\right)=\theta-4 \arctan (X), \\
\alpha^{\prime}\left(l_{s}^{-}\right) & =\alpha^{\prime}\left(l_{s}^{+}\right)=\frac{4 X}{r\left(1+X^{2}\right)} .
\end{aligned}
$$

The continuity of the curvature $\alpha^{\prime}$ is equivalent to the absence of point torque at $s=l_{s}$. Its derivative $\alpha^{\prime \prime}$ is actually also continuous at the end of the cohesive zone, because no point force is applied there. The latest continuity relation is however already imposed by the continuity of $\alpha$ associated to Eq. 7 in which the term multiplying $\bar{\sigma}$ disappears at $s=l_{s}$.

Similarly, continuity relations at $s=0$ are

$$
\begin{aligned}
\alpha\left(0^{-}\right) & =\alpha\left(0^{+}\right)=0, \\
\alpha^{\prime}\left(0^{-}\right) & =\alpha^{\prime}\left(0^{+}\right)=0 .
\end{aligned}
$$


Using Eq. 7 and the boundary conditions (10) and (11) one can compute the value taken by the energy release rate $G=(1-\cos \theta) F / b$ during steady-state propagation according to this model

$G=\bar{\sigma}\left(a_{f}-a_{0}\right)$.

$G$ is actually equal to the work (per unit peeled surface) of the cohesive forces during the stretching of the adhesive from its initial thickness $a_{0}$ up to the maximum fibril length $a_{f}=a\left(s=l_{s}\right)$ at debonding. This relation, which tells that the work of the peeling force $\vec{F}$ is fully used to stretch the adhesive material, actually simply accounts for the energy conservation in the bending deformation of the tape backing during steady-state peeling. Note that, in contrast, in the simple elastica model of Eq. 4 with no cohesive zone, bending energy conservation can only be enforced thanks to the introduction of a curvature discontinuity at $s=0$ that acts as an energy sink

$G=\frac{E I}{2 b}\left[\alpha^{\prime}\left(0^{+}\right)^{2}-\alpha^{\prime}\left(0^{-}\right)^{2}\right]$.

We can finally rewrite the whole system describing the tape profile in the cohesive zone $\left(0 \leq s \leq l_{s}\right)$, with the boundary conditions

$$
\begin{aligned}
\alpha(0) & =0, \\
\alpha\left(l_{s}\right) & =\theta-4 \arctan (X), \\
\alpha^{\prime}\left(l_{s}\right) & =\frac{4 X}{r\left(1+X^{2}\right)},
\end{aligned}
$$

${ }^{4}$ Multiplying Eq. 7 by $\alpha^{\prime}$ and integrating from 0 to $l_{s}$ yields

$$
\begin{aligned}
\frac{8 F X^{2}}{\left(1+X^{2}\right)^{2}} & +F[\cos (4 \arctan X)-\cos \theta] \\
& -\bar{\sigma} b \int_{0}^{l_{s}}\left[\cos (\alpha(s)) \int_{s}^{l_{s}} \sin (\varphi(t)) d t\right] \alpha^{\prime}(s) d s \\
& -\bar{\sigma} b \int_{0}^{l_{s}}\left[\sin (\alpha(s)) \int_{s}^{l_{s}} \cos (\varphi(t)) d t\right] \alpha^{\prime}(s) d s=0 .
\end{aligned}
$$

After trigonometric simplifications of $\cos (4 \arctan (X))$ and integration by parts of the two integrals, we get

$$
\begin{aligned}
& \frac{F}{b}(1-\cos \theta)= \\
& \quad \bar{\sigma} \int_{0}^{l_{s}}\{\sin (\alpha(s)) \sin (\varphi(s))+[1-\cos (\alpha(s))] \cos (\varphi(s))\} d s .
\end{aligned}
$$

The integrand can be rewritten as

$$
\begin{aligned}
\sin \varphi \sin \alpha+(1-\cos \alpha) \cos \varphi & =\frac{\left(y+a_{0}\right) \frac{d y}{d s}+(s-x) \frac{d s-d x}{d s}}{\sqrt{\left(y+a_{0}\right)^{2}+(s-x)^{2}}} \\
& =\frac{d a(s)}{d s}
\end{aligned}
$$

where $a(s)=\sqrt{\left(y+a_{0}\right)^{2}+(s-x)^{2}}$ is the length of the fibril attached to the tape backing at curvilinear position $s$. This finally leads to Eq. 12 . One can also demonstrate Eq. 12 by computing the work associated to a small advance of the peeling front (see Online Resource 2). associated to the master equation

$$
r^{2} \alpha^{\prime \prime}+\sin (\theta-\alpha)-\frac{1-\cos \theta}{a_{f}-a_{0}} \int_{s}^{l_{s}} \sin [\alpha(s)+\varphi(t)] d t=0,
$$

where the maximum fibril length $a_{f}$ is given by

$$
a_{f}=\sqrt{\left(\int_{0}^{l_{s}}(1-\cos \alpha(s)) d s\right)^{2}+\left(a_{0}+\int_{0}^{l_{s}} \sin \alpha(s) d s\right)^{2}} .
$$

Note that, since Eq. 12 was used in Eq. 14 to replace $\bar{\sigma}$, one of the initial boundary conditions has become redundant: we have therefore removed the $\alpha^{\prime}(0)=0$ boundary condition from the system.

This system contains an integro-differential equation with derivatives up to the second order, plus one unknown, $X$, requiring therefore three boundary conditions, which are provided by Eq. 13. We can solve this problem for different sets of values for $\theta, r, a_{0}$ and $l_{s}$, the only parameters it depends on. Note that this problem does not depend explicitly on $\bar{\sigma}$ anymore. However, $\bar{\sigma}$ can be retrieved via Eq. 12 , using $a_{f}\left(l_{s}\right)$ determined from the tape profile and the fact $G$ is determined by $F$ and $\theta$ (see Eq. 1), which can be easily and precisely measured.

To solve the set of Eqs. 13 and 14, we normalize all lengths by $r$ and we sample the interval $s / r \in\left[0: l_{s} / r\right]$ over $N$ points (typically several hundreds to thousands). We replace $\alpha^{\prime \prime}$ by its midpoint method estimate, and $\alpha(1), \alpha(N-1)$ and $\alpha(N)$ by their expressions according to (13). We then use the Matlab fsolve routine to find the values of $X$ and $\alpha(2) \ldots \alpha(N-2)$ that minimize the set of $N-2$ equations obtained from Eq. 14. All lines in this system contain the term $\sin (\theta-\alpha)$, which is of order of magnitude 0.1 to 1 , enabling an objective criterion for the minimization procedure: the system is considered to be solved when all lines are very small compared to 0.1 (in practice when the maximum term is smaller than $10^{-6}$ ). We use as initial guesses for $X$ and $\alpha(i)$ the analytical solutions of the approximation of Eq. 14 for small angles $\alpha$ (see Online Resource 2).

Fig. 5 reports numerical solutions of the system of equations 13-14 for peeling angles $\theta$ and cohesive zone size ratios $l_{s} / r$ typical of our experiments. As expected, the solution converges towards the simple elastica profile Eq. 4 at small $l_{s} / r$ values. We can also observe that the curvature (slope of $\alpha(s)$ ) goes from 0 at $s=0$ up to a maximum included in the cohesive zone, before decreasing back towards 0 at $s=\infty$.

Computing the dimensional profile $\alpha(s)$ (and thus the $y(x)$ profile by integration) eventually requires to know $r$ and $l_{s}$, as well as the curvilinear abscissa $s_{0}$ along the tape 

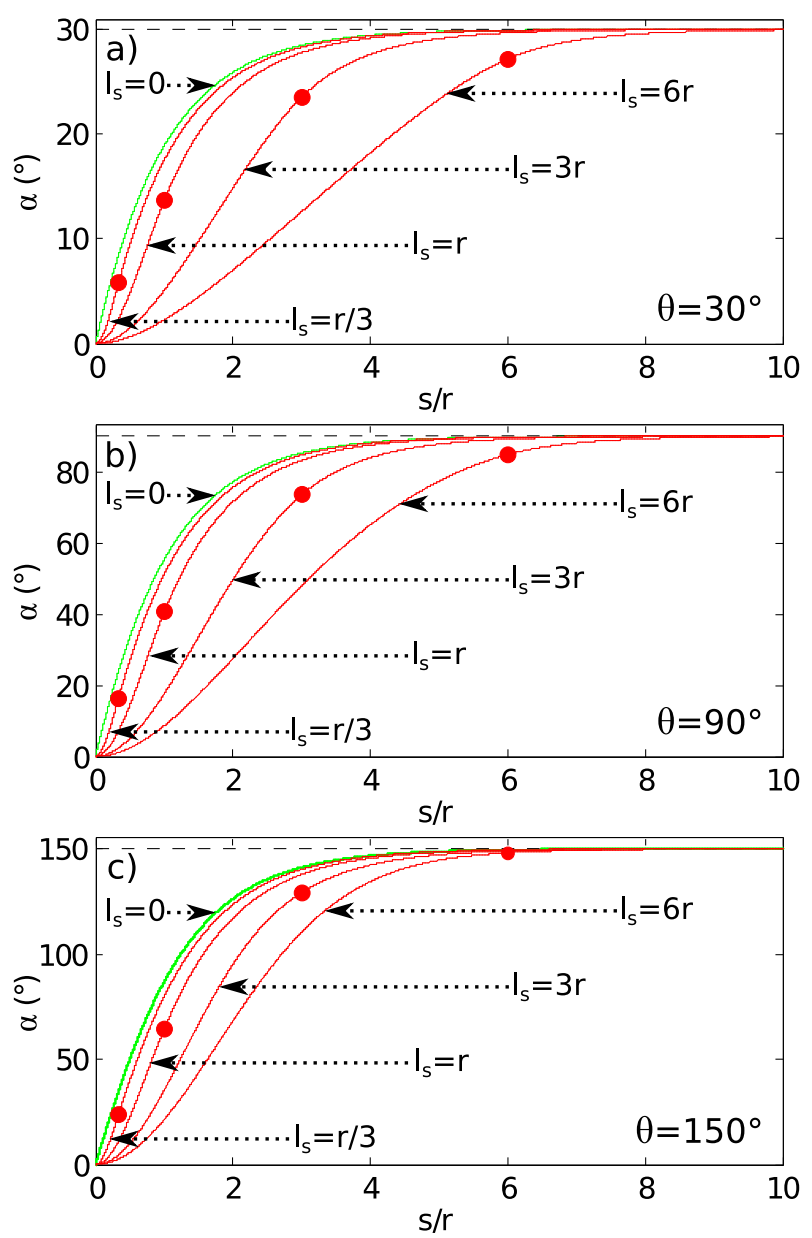

Fig. 5 Theoretical profiles of elasticas with cohesive zones of different sizes $l_{s}$ (the ends of which are shown by red disks) and for different peeling angles $\theta$. We normalize the curvilinear abscissa $s$ by the typical radius of curvature $r=\sqrt{E I / F}$. The considered values for the ratio $l_{s} / r$ are typical of our experiments (Figs. 1 and 2).

at which the debonding region begins (i.e. the starting point of the local reference frame to compute the curvilinear abscissa, taken as 0 for the sake of simplicity in Eqs. 6 to 14). For each experimental profile of the tape backing, $r=$ $\sqrt{E I / F}$ is first obtained from the fit of $\alpha(s)$ outside of the debonding region by the simple elastica prediction (9) (leaving the constant $X$ as an unused parameter at this step). The whole experimental profile $\alpha(s)$ (including the debonding region) can finally be fitted by the system of equations (1314) using $l_{s}$ and $s_{0}$ as the only fitting parameters ${ }^{5}$.

\footnotetext{
${ }^{5}$ Once we know $\alpha(s), l_{s}$ and $s_{0}, a_{f}$ can be determined by Eq. 15 . Curvilinear abscissa is eventually redefined as $s \rightarrow s-s_{0}$ after the fit, in order to set the beginning of the cohesive zone to $s=0$.
}

\section{Results and discussion}

\subsection{Comparison between model and experimental profiles}

The fitting procedure described in Sec. 4 has been first systematically applied to all the individual frames of the experimental movies, for all couples of control parameters $(\theta, V)$.

Both the radius of curvature $r$ and the size of the cohesive zone $l_{s}$, which are the two valuable raw products of the fitting procedure, are found to be very stable during each movie (see the Online Resource 1), corresponding to the peeling of typically several hundreds of $\mu \mathrm{m}$ to several $\mathrm{cm}$, depending on $V$. More precisely, we observe typical slow variations of 5 to $15 \%$ for these two lengths during a peeling experiment. Besides, when large portions of fibrils debond at once, the tape backing profile and thus the fitted value of $l_{s}$ do not change significantly (2\% for the strongest event at time $t=4.6 \mathrm{~s}$ in the movie in the Online Resource 1, with a cumulated variation in $l_{s}$ of $13 \%$ along the entire movie). On the other hand, the extent of the region where the fibrils are observed on the visible side presents a scatter of $\pm 20 \%$. This last observation is related to the fact the only visible fibrils in the movie are the ones on the side of the tape observed by the camera, while the tape backing profile is the result of the influence of the debonding region over the whole tape width $b$. This eventually makes our fitting procedure a more accurate and representative tracer of the geometry of the debonding region over the whole tape width than the optical observations of the fibrils on the side view, which represent a limited set of fibrils that are close to the side of the tape. A detailed analysis reveals that the average size of the debonding region observed on the tape side presents a bias going from $-20 \%$ to $+20 \%$ with respect to the cohesive zone length extracted from the fitting of the backing profile for $\theta$ ranging from $30^{\circ}$ to $120^{\circ} .^{6}$ The extent of the debonding region on the tape side tends to be shorter than at the center of the tape when the peeling angle is less than $90^{\circ}$ and longer for peeling angles larger than $90^{\circ}$. This bias reveals an interesting edge effect that is not at the core of the present investigation. In the rest of the manuscript we will focus on the estimations derived from the bending profile, which are not affected by this edge effect.

Because of the small fluctuations of the detected tape backing profiles (due in particular to dusts on the tape or tape imperfections) and of the lengths $r$ and $l_{s}$ extracted from them, we have applied as a second step the fitting procedure to profiles averaged over all images in a movie, which enables to considerably increase the signal-to-noise ratio. The results of this fitting procedure is in excellent agreement with the experimental profiles, as can be seen in Figs. 2 and 3 , including in the debonding region. This agreement con-

6 The bias gets larger for the largest angle $\theta=150^{\circ}$, which will be discussed later. 
firms the relevance of the model of elastica combined with a constant stress cohesive zone, which catches the main features of the tape profile using only few mechanical ingredients : a mean stress $\bar{\sigma}$ oriented along the fibrils over a region of length $l_{s}$.

\subsection{Cohesive stress and maximum fibril extension}

In the model developed in Sec. 4, energy dissipation during peeling occurs because of the loss of the work expended to stretch the adhesive material up to debonding, which brings the following expression for the effective fracture energy of peeling

$\Gamma=\bar{\sigma}\left(a_{f}-a_{0}\right)$.

This work done against the cohesive stress $\bar{\sigma}$ is partially dissipated by viscosity during the stretching of the adhesive, the rest being stored in the adhesive elastic energy and eventually lost at debonding by "elastic hysteresis" (Villey et al. 2015).We remark that the transient kinetic energy developed by the fibrils after debonding is also dissipated by viscosity during the fibrils collapse, but in any case this evolution is not coupled to the peeling mechanics since the broken fibrils are not bridging the backing to the substrate anymore.

In this section we use the results of the fitting of the experimental tape profiles to access the two parameters controlling energy dissipation during peeling (according to the cohesive zone model): (i) the average stress $\bar{\sigma}$ in the cohesive zone and (ii) the fibrils extension $a_{f}-a_{0}$ at the onset of debonding, which are reported in Fig. 6.

Fig. 6(a) reports the evolution of the adherence energy $\Gamma(\theta, V)=\bar{\sigma}\left(a_{f}-a_{0}\right)$ as a function of the peeling angle $\theta$ for peeling velocity $V=0.1 \mathrm{~mm} \mathrm{~s}^{-1}$. As reported in previous experiments on the same adhesive-substrate joint (Villey et al. 2015), the adherence energy is found to be a regularly increasing function of the peeling angle $\theta$. Assuming the cohesive zone model developed in Sec. 4 and considering Figs. 6 (b) and (c), this angular dependency seems to be mainly due to the fibril elongation $a_{f}-a_{0}$ that is found to be clearly increasing with $\theta$, while the average stress in the cohesive zone $\bar{\sigma}$ is displaying weaker trends. If all angles are considered, the average stress decreases by a factor 1.7 while the fibril elongation increases by a factor 3.7 .

We should remark that when limiting to the angles up to $120^{\circ}, \bar{\sigma}$ is almost constant and all the increase in $\Gamma$ is due to the increase in $a_{f}-a_{0}$. In fact, the case of $\theta=150^{\circ}$ presents some peculiarities compared to all other experimental conditions studied in this paper: at this large angle (see Fig. 2e), the detected sizes $l_{s}$ of the cohesive zone seem systematically larger (by typically 30-60\%) than the visible fibrils region (keeping in mind that those may not be representative of all fibrils along the tape width). Moreover, a careful examination of the $\alpha(s)$ profiles at this large angle reveals a systematic overshoot of $1^{\circ}$ to $2^{\circ}$ in the local angle $(\alpha>\theta$ locally, a feature too small to be visible in Fig. 2). Such an overshoot can be attributed to the occurrence of some plastic deformation in the tape backing, which should add a residual curvature to the tape profile. If plasticity happens, it should indeed be at large peeling angles (Gent and Hamed 1977; Derail et al. 1997; Derail et al. 1998), where the tape curvature is large. This is consistent with a simple everyday life test: an office tape remains twisted after being peeled when peeling proceeds at a large angle. However, the values for $a_{f}-a_{0}$ and $\bar{\sigma}$ obtained at $\theta=150^{\circ}$ remain of the same order as the ones obtained at smaller angles, and the fitted profiles remain in good agreement with the experimental ones even at this large angle: this indicates that the results our model produces at very large peeling angles may be conserved, while considered with precaution.

Figs. 6 (d-f) report the same three quantities, $\Gamma, \bar{\sigma}$ and $a_{f}-a_{0}$, as a function of the peeling velocity $V$ for $\theta=$ $90^{\circ} . \Gamma$ and $\bar{\sigma}$ follow power laws of $V$ with close exponents ( 0.20 and 0.27 respectively): contrary to the angular dependency, we observe that most of the increase of the adherence energy $\Gamma=\bar{\sigma}\left(a_{f}-a_{0}\right)$ with the peeling velocity is due to the increase of the cohesive zone stress $\bar{\sigma}$ and that it is only slightly affected by a weak decrease of the fibril extension $a_{f}-a_{0}$ with $V$. Moreover, the values reported for $\bar{\sigma}$ in Figs. 6 (b) and (e) are consistent with what is expected for the plateau stress of such acrylic-based PSA (Lindner et al. 2004; Lindner et al. 2006).

In the present work, we have studied only two line cuts $\left(V=0.1 \mathrm{~mm} \mathrm{~s}^{-1}\right.$ and $\left.\theta=90^{\circ}\right)$ in the experimental parameters plane $(\theta, V)$. Reference (Villey et al. 2015) reports measurements of the peeling adherence energy $\Gamma$ for the same PSA over a wider range of parameters $(\theta, V)$. The data reported in (Villey et al. 2015) actually revealed that the dependencies of $\Gamma$ with $V$ and $\theta$ were almost separable, i.e. that $\Gamma(\theta, V) \simeq f(\theta) g(V)$. In light of our present experiments, this separability can be justified by the fact that for this adhesive the dependence of $\bar{\sigma}$ on the angle $\theta$ is barely detectable, and in any case is weak compared to the one of $a_{f}(\theta)$, and conversely that the decreasing trend of $a_{f}(V)$ is weak compared to the increasing trend of $\bar{\sigma}(V)$, so that we can write

$$
\begin{aligned}
\Gamma(\theta, V) & =\bar{\sigma}(\theta, V) \times\left[a_{f}(\theta, V)-a_{0}\right] \\
& \approx \bar{\sigma}(V) \times\left[a_{f}(\theta)-a_{0}\right] .
\end{aligned}
$$

In addition to the average stress and the maximum elongation, another key rheological parameter can be extracted from our treatment of peeling tests: the average strain rate

$\overline{\dot{\varepsilon}}=\frac{1}{l_{s}} \int_{0}^{l_{s}} \dot{\varepsilon}(s) d s=\frac{V}{l_{s}} \ln \left(\frac{a_{f}}{a_{0}}\right)$. 

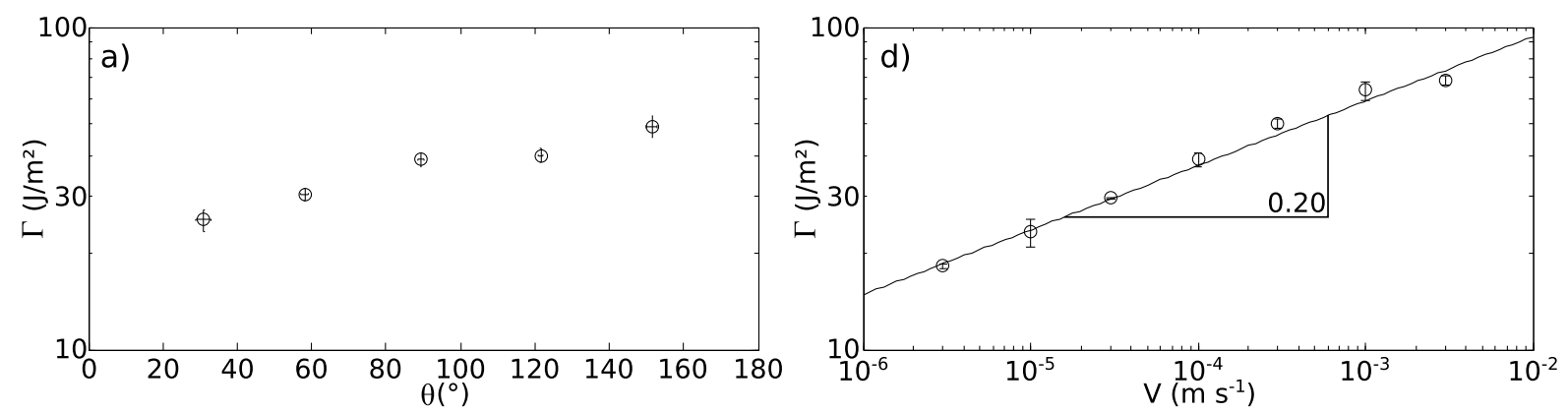

b)
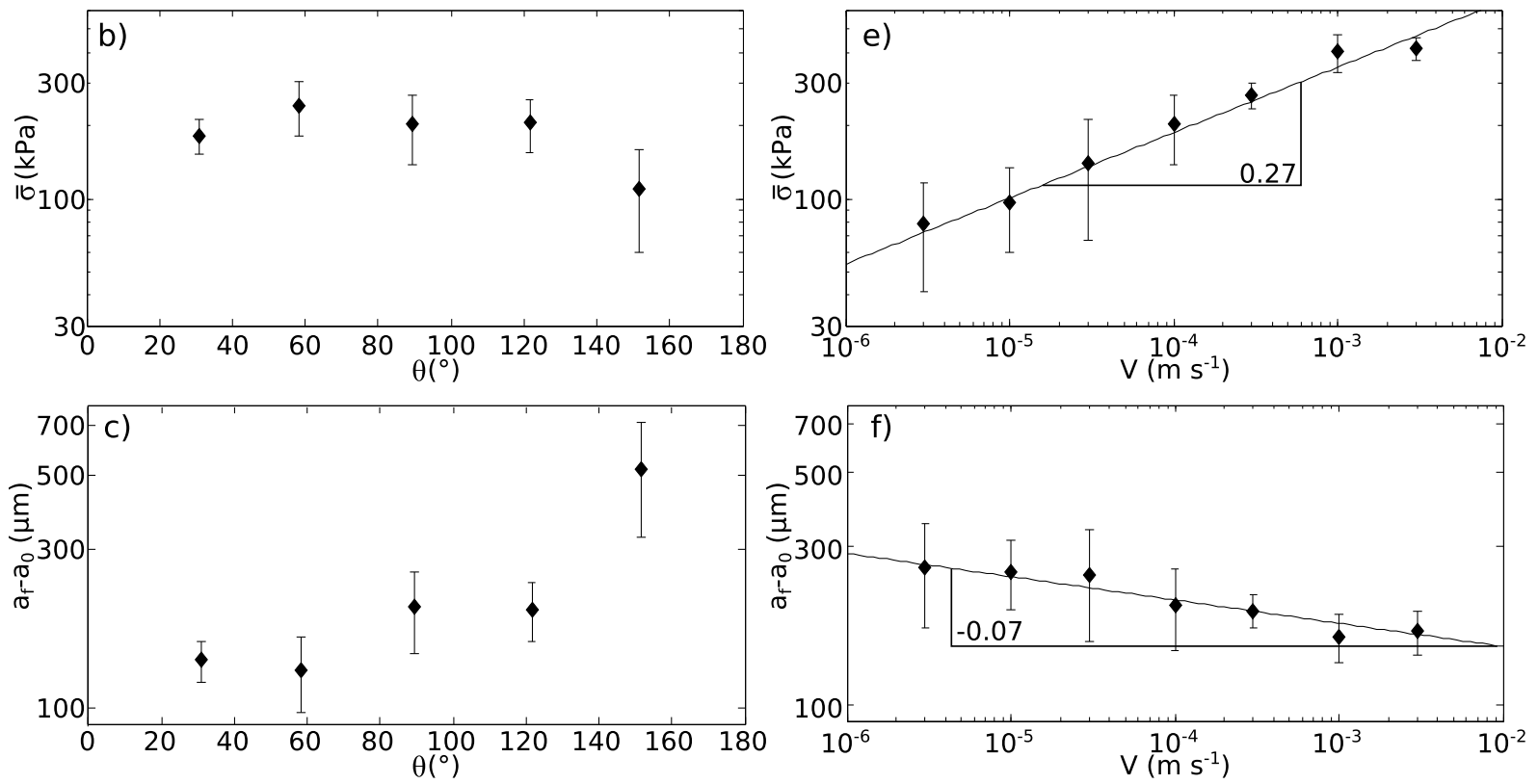

Fig. 6 Adherence energy $\Gamma$, average stress $\bar{\sigma}$ in the cohesive zone and fibril elongation at debonding $a_{f}-a_{0}$ for peeling experiments at $V=$ $0.1 \mathrm{~mm} \mathrm{~s}^{-1}$ and different peeling angles $\theta(\mathrm{a}-\mathrm{c})$ and at $\theta=90^{\circ}$ and different peeling velocities $V(\mathrm{~d}-\mathrm{f}) . \Gamma$ is measured through the peeling force $F$, using Eqs. 1-2. $a_{f}$ is obtained from the fit of the tape backing profiles by Eq. 15. $\bar{\sigma}$ is further obtained using Eq. 16.

at which the adhesive material is strained in the debonding region $\left(\dot{\varepsilon}(s)\right.$ is the local logarithmic strain). ${ }^{7}$ Fig. 7(a) shows that $\overline{\dot{\varepsilon}}$ is increasing by a factor less than 1.8 for $\theta$ ranging from $30^{\circ}$ to $150^{\circ}$ for $V=0.1 \mathrm{~mm} \mathrm{~s}^{-1}$, a factor comparable to the variations of the local strain rate $\dot{\varepsilon}(s)$ over the cohesive zone. In parallel, we see in Fig. 7(b) that $\overline{\dot{\varepsilon}}$ increases by more than three orders of magnitude with $V$ increasing from $3 \cdot 10^{-6}$ to $3 \cdot 10^{-3} \mathrm{~m} \mathrm{~s}^{-1}$ for $\theta=90^{\circ}$. This last dependency

\footnotetext{
7 The evaluation of the average strain rate requires the calculation of the temporal dependence of the length $a(t)$ of a typical fibril during the peeling. We should thus operate a change of reference frame from the one of the laboratory (where the bending profile is steady and identified by our measurements of $\alpha(s)$ ) to the local reference frame of the substrate. The evolution of the bending profile in time in the local reference frame should be described by another slope function $\beta(u, t)$ expressed in terms of the material curvilinear abscissa $u$. Since the substrate is moving at the same velocity as the peeling velocity $V$, the two bending functions are related by $\alpha(u+V t)=\beta(u, t)$. From the logarithmic strain $\varepsilon(s)=\ln \left(a(s) / a_{0}\right)=\ln \left(\sqrt{(s-x)^{2}+\left(y+a_{0}\right)^{2}}\right)-\ln \left(a_{0}\right)$ of the fibril attached at position $s$ in the laboratory reference frame, we can then compute the local strain rate as $\dot{\varepsilon}(s)=\frac{1}{a(s)} \frac{d a}{d s} \frac{\partial s}{\partial t}=\frac{V}{a(s)} \frac{d a}{d s}$, which after integration results in Eq. 18.
}

is well accounted for by a nearly linear power law $\overline{\dot{\varepsilon}} \sim V^{1.06}$. The fact the relationship between $\overline{\dot{\varepsilon}}$ and $V$ is almost linear means that the factor $\ln \left(a_{f} / a_{0}\right) / l_{s}$ in Eq. 18 is almost independent of the peeling velocity. This result is related to the fact the lengths $l_{s}$ and $a_{f}$ follow very small exponent power law of $V\left(l_{s} \propto V^{-0.08}\right.$ and $\left.a_{f} \approx a_{f}-a_{0} \propto V^{-0.07}\right)$. In fact, $l_{x}$ and $l_{y}$ also follow power laws of $V$ with very close small exponents $(-0.08$ and -0.075 respectively): the cohesive zone is self-similar to the first order, with just a weak downscaling when $V$ increases.

Since one can expect a direct influence of the strain rate of the adhesive material on the level of stress, we also report in Fig. $8 \bar{\sigma}$ as function of $\overline{\dot{\varepsilon}}$ for all studied couples of control parameters $(\theta, V)$. These data reveal that $\bar{\sigma}$ nearly follows a power law $\bar{\varepsilon}^{1 / 4}$ when $V$ is varied, except for the data acquired at $\theta=150^{\circ}$ and $V=0.1 \mathrm{~mm} \mathrm{~s}^{-1}$, which depart from all other data presented in this paper, probably due to incipient plastification of the backing as discussed before. For the other angles studied at this same velocity, the influence of changing $\theta$ does not dramatically move away the data from 

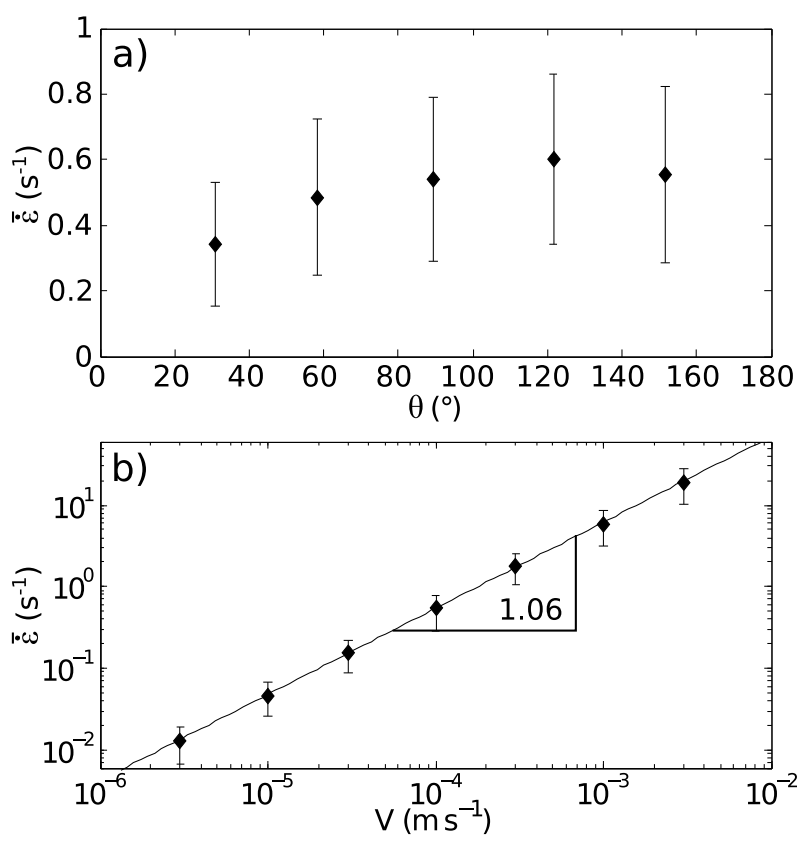

Fig. 7 Average strain rate $\overline{\dot{\varepsilon}}$ in the cohesive zone, obtained from the fitted tape backing profiles (using the model of Sec. 4) and from Eq. 18. $\overline{\dot{\varepsilon}}$ is represented (a) as a function of $\theta$ for $V=0.1 \mathrm{~mm} \mathrm{~s}^{-1}$ and (b) as a function of $V$ for $\theta=90^{\circ}$. The error bar is the standard deviation of $\dot{\varepsilon}(s)$ in the cohesive zone.

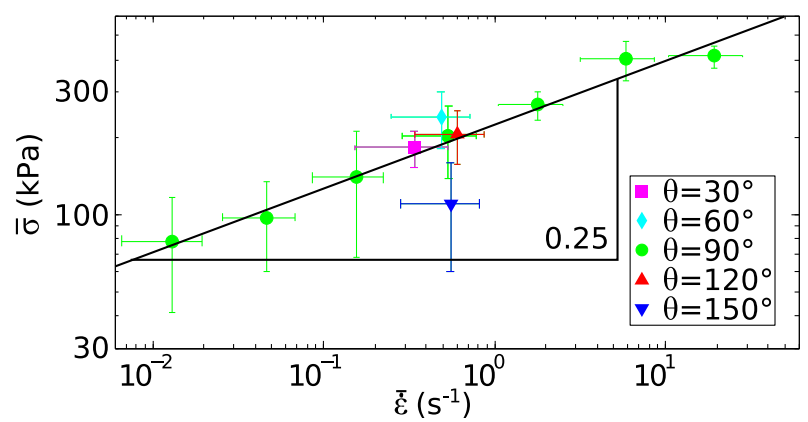

Fig. 8 Average stress $\bar{\sigma}$ (from Figs. 6(b) and (e)) as a function of the average strain rate $\overline{\dot{\varepsilon}}$ (from Fig. 7) in the debonding region, for all the tested couples $(\theta, V)$ of control parameters.

the main power law, in agreement with the limited influence of $\theta$ on $\bar{\sigma}$ and on $\overline{\dot{\varepsilon}}$ (compared to the one of $V$ ). Therefore, one can say that the typical stress in the debonding region $\bar{\sigma}$ is to the first order controlled by the value of the mean strain rate $\overline{\dot{\varepsilon}}(\theta, V)$ to the power $\sim 1 / 4$, at least for peeling angles up to $120^{\circ}$. At these angles, the role of $\theta$ in the relation between $\bar{\sigma}$ and $\overline{\dot{\varepsilon}}$ remains unresolved, but it is undoubtedly of second order.

A last parameter interesting to look at is the angle $\varphi_{f}=$ $\arctan \left(\frac{a_{0}+l_{y}}{l_{s}-l_{x}}\right)$ between the last fibril of length $a_{f}$ and the substrate, as reported in Fig. 9. We should highlight here that the values we report for $\varphi_{f}$ result from the fit of the tape profile by the cohesive zone model and are not obtained from direct observations of the fibrils inclination (which would
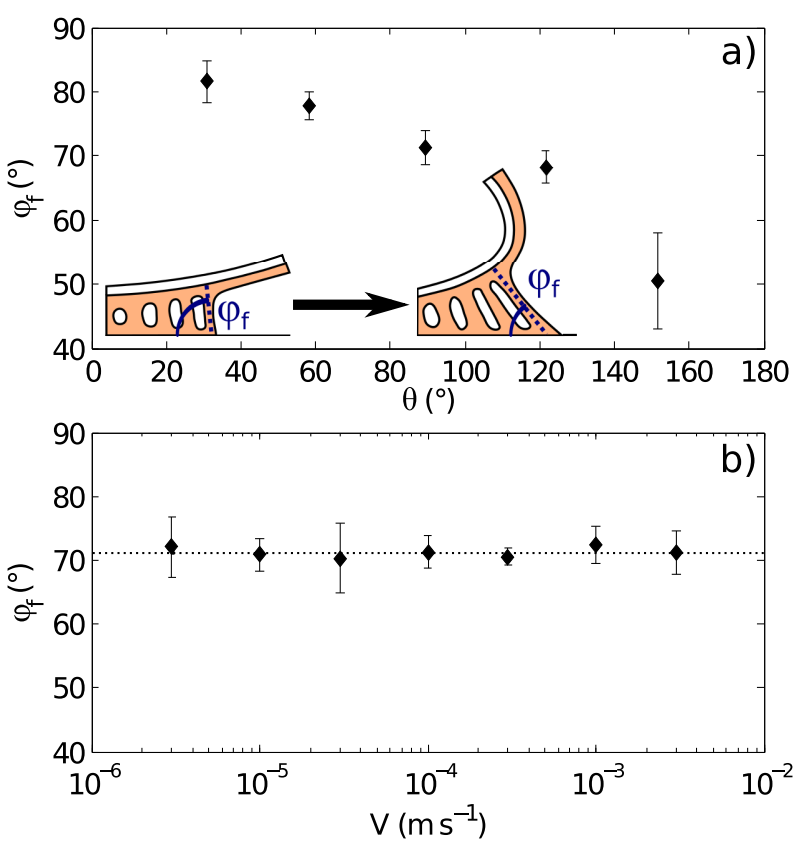

Fig. 9 "Angle at debonding" $\varphi_{f}$ between the substrate and the longest fibril (about to debond), (a) for $V=0.1 \mathrm{~mm} \mathrm{~s}^{-1}$ and different peeling angles $\theta$ and (b) for $\theta=90^{\circ}$ and different peeling velocities $V . \varphi_{f}=$ $\arctan \left(\frac{a_{0}+l_{y}}{l_{s}-l_{x}}\right)$ is obtained through the fit of experimental profiles by the model of Sec. 4.

nevertheless be closely matching for $\left.\theta \leq 120^{\circ}\right)$. We find that $\varphi_{f}$ is remarkably constant with $V$ (at least at $\theta=90^{\circ}$ ), which is consistent with the self-similar downscaling of the cohesive zone with increasing $V$. In parallel, $\varphi_{f}$ decreases from about $80^{\circ}$ at $\theta=30^{\circ}$ down to approximatively $50^{\circ}$ for the highest peeling angle $\theta=150^{\circ}$ (where the angle of the visible side fibrils with the substrate is around $65-75^{\circ}$, a value sensibly higher than the one obtained with our model fit, which displays some limitations at very large peeling angles, as abovementioned). These features suggest a correlation between the maximum fibril elongation $a_{f}-a_{0}$ and their inclination $\varphi_{f}$, which we call the "angle at debonding" of the fibrils.

Understanding the debonding criterion for a highly stretched soft fibril from a substrate is clearly a key to modeling the adherence energy $\Gamma(V, \theta)$ of PSAs. While this remains a very subtle unsolved problem problem (Yarusso 1999; Villey et al. 2015; Creton and Ciccotti 2016) and is out of the scope of the present work, the availability of our measurements of $a_{f}, \overline{\dot{\varepsilon}}$ and $\varphi_{f}$ is very promising for future investigations on custom PSA with controlled rheology and surface conditions.

\subsection{Beyond the cohesive zone model}

The good agreement between the imaged tape profiles and the fits suggests that extracting more information from these 
profiles than an average stress in the debonding region may not be an easy task. Obtaining more refinements in the stress distribution indeed implies to look at the minute differences between the fitted profiles and the experimental ones. Notice that the exact stress distribution can theoretically be accessed by computing the fourth derivative $d y^{(4)} / d x^{4}$ of the tape profile (Love 1944; Niesiolowski and Aubrey 1981). However, since the typical size of the debonding region is in the hundreds of $\mu \mathrm{m}$ range, even getting a spatial stress distribution with a few resolved values is difficult: getting the changes in $d y^{(4)} / d x^{4}$ with a resolution as low as several tens of $\mu \mathrm{m}$ would require a defectless tape (no dusts or scratches) at the $\mu \mathrm{m}$ scale, which is hardly reachable in practice. The same issue led Niesiolowski and Aubrey to upscale the size of their adhesive tape in order to apply this method (using derivatives of the tape profile up to the fourth order to compute the forces acting on the tape backing, see appendix 3 in (Niesiolowski and Aubrey 1981)). Eventually, it seems that only a few average stress values can be inferred at best from the whole tape profile.

A thorough examination of the minute differences between the experimental profiles and their fit by our model does nevertheless reveal one systematic feature that can be used for further refinements, enabling to obtain more information about the actual stress distribution. A zoom at the very beginning of the debonding region (see Fig. 10) indeed reveals a small zone where the tape displacement normal to the substrate $y(s)$ is negative, corresponding to a local vertical compression of the adhesive layer.

This can be explained by considering that, before reaching a plateau value $\bar{\sigma}$, the stress in the adhesive has to build up from zero. The simplest model for this increase consists in considering the linear elastic behaviour of the adhesive at small strain, as proposed by Kaelble (Kaelble 1959; Kaelble 1960), which results in such a compression zone before the adhesive is significantly stretched. Kaelble actually modeled the adhesive layer by a linear rate-dependent elastic foundation which, in our case, can be relevant to describe the initial part of the debonding region (before cavitation and fibrillation) where the adhesive is not too much strained. In this zone, Kaelble predicts the following tape profile:

$$
\begin{aligned}
& y=y_{0} e^{\frac{x}{\lambda_{\beta}}}\left[\cos \left(\frac{x}{\lambda_{\beta}}\right)+K \sin \left(\frac{x}{\lambda_{\beta}}\right)\right] \text {, } \\
& \text { with } \lambda_{\beta}=\sqrt[4]{\frac{4 E I a_{0}}{Y b}} \text {, } \\
& \text { and } K=1-\frac{\lambda_{\beta} \sin \theta}{r \sqrt{2-2 \cos \theta}-0.5 h \cos \theta+\lambda_{\beta} \sin \theta} \text {. }
\end{aligned}
$$

In this equation, $h$ is the backing thickness and $Y$ is the adhesive effective tensile modulus (larger than the adhesive Young's modulus because of incompressibility and confinement, see discussion in (Villey et al. 2015)).

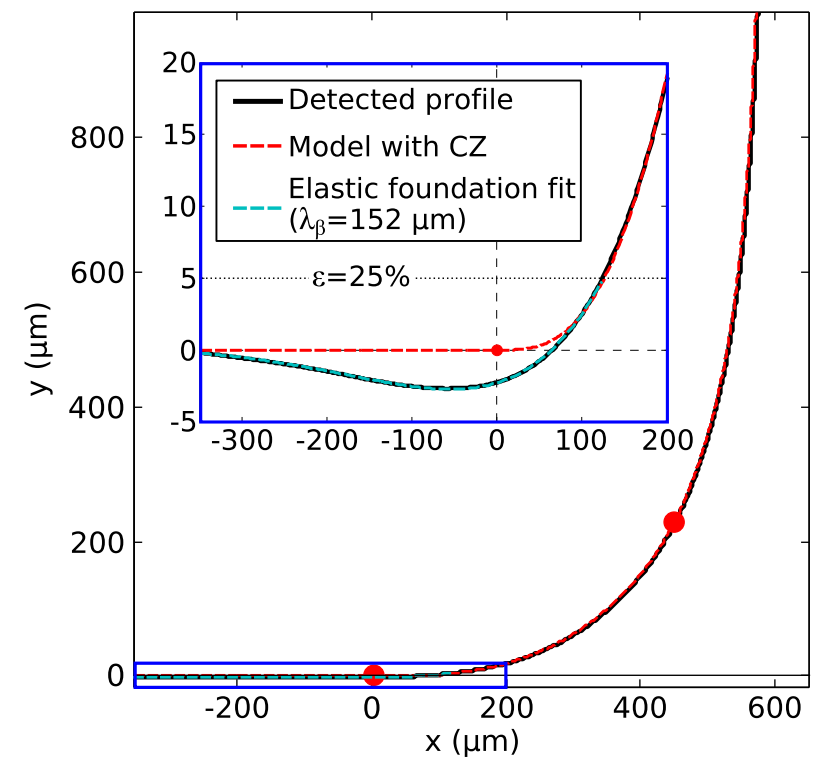

Fig. 10 The inset represents the zoom of the basal region (blue rectangular box $)$ of an experimental profile $\left(\theta=90^{\circ}, V=100 \mu \mathrm{m} \mathrm{s}^{-1}\right)$ after averaging over 540 frames. The entire profile was fitted using the cohesive zone (CZ) model of Eqs. 13 and 14 (red profile, with the $\mathrm{CZ}$ beginning at $s=0$ ); the zone where the backing displacement $y$ corresponds to an adhesive deformation smaller than $25 \%$ (i.e. $|y|<a_{0} / 4=5 \mu \mathrm{m}$ ) was fitted using the linear elastic foundation model of Eq. 19, providing an estimate of $\lambda_{\beta}$ (and thus of $Y$ ) as a fitting parameter.

We should remark that in Kaelble's model the position $x=0$ corresponds to the location where the normal stress $\sigma(x)=Y y / a_{0}$ in the adhesive reaches a maximum value $\sigma_{0}$, which is also the location of adhesive debonding when $\sigma_{0}$ reaches a critical value (we do not discuss the shear stress distribution here because it is not relevant for the peeling angles discussed in this paper, see (Villey et al. 2015)). The same equation 19 remains however valid and can be combined with our cohesive zone model if we assume that the position $x=0$ corresponds to the transition between the linear elastic zone (where the normal stress builds up in the confined adhesive) and the cohesive zone (where the stress reaches saturation in the fibrillated adhesive), i.e. that $Y y_{0} / a_{0}=\bar{\sigma}$.

A first refinement of our model could thus simply consist in combining an elastic foundation with a cohesive zone, where in the foundation $(-\infty<s \leq 0)$ the adhesive response is linear elastic $\left(\sigma(x)=\sigma(s)=Y y / a_{0}\right)$ and where in the cohesive zone $\left(0 \leq s \leq l_{s}\right)$ it is plastic-like $(\sigma(s)=\bar{\sigma})$. In such a model, the adherence energy is given by

$\Gamma=a_{0} \frac{\bar{\sigma}^{2}}{2 Y}+\bar{\sigma}\left(a_{f}-a_{0}-y_{0}\right)$,

which is simply the sum of the work to first stretch the adhesive in the elastic foundation and then in the cohesive zone up to debonding.

Such a model combining the elastic foundation and the cohesive zone certainly offers a better description of the 
stress distribution within the adhesive, but its relevance to explain energy dissipation during peeling should be further examined. To do so, we estimate the importance of the elastic foundation contribution to the adherence energy in Eq. 20 compared to the contribution of the cohesive zone. We thus need estimates of $\bar{\sigma}$ and $Y$, the former being given by fitting the whole profile with the model of Sec. 4 and the latter by fitting the detected profile in the small strain zone by Eq. 19: in this zone, the characteristic length $\lambda_{\beta}$ of the spatially damped oscillation of the tape profile can be fitted, giving access to $Y=b \lambda_{\beta}^{4} / 4 E I a_{0}$. Practically, the tape profile was fitted using Eq. 19 with $K, y_{0}$ and $\lambda_{\beta}$ as independent fitting parameters, as it is done for example in Fig. 10.

The systematic fitting of the averaged experimental profiles provides measurements of $\lambda_{\beta}$, which reveal $Y$ values in the hundreds of $\mathrm{kPa}$ to several MPa range: this is larger than the several tens of $\mathrm{kPa}$ expected for the unconfined adhesive (Amouroux et al. 2001), but it can be explained by the confinement effect: an incompressible material uniaxially stretched from an initial confined geometry has a stiffer response than in an unconfined stretching test.

The contribution of the elastic foundation in the overall energy budget is slightly decreasing with $\theta$ and globally increasing with $V$. More precisely, $a_{0} \bar{\sigma}^{2} /(2 Y \Gamma)<5 \%$ in all our experiments, except at $V=0.3 \mathrm{~mm} \mathrm{~s}^{-1}$ where it is around $8 \%$ and at $V=3 \mathrm{~mm} \mathrm{~s}^{-1}$ where it is around $30 \%$.

This means that the experiments presented in this paper correspond to the case where the stress plateau dominates the dissipation in the stretched adhesive, and that the elastic foundation zone can safely be neglected both in the energy budget and when estimating the level of the stress plateau, except maybe at the highest tested peeling velocity $V=3 \mathrm{~mm} \mathrm{~s}^{-1}$. The model of Sec. 4 is however expected to become less relevant at higher velocities, where the maximum fibrils extension may become small enough so that the contribution of the elastic foundation zone becomes important in the overall work used to stretch the adhesive. The more refined model briefly described in this section could therefore be very useful to extract relevant local rheological parameters and to explain energy dissipation at larger peeling velocities than in the present investigation, in order to give an overall complete picture of the peeling mechanics of soft confined layers.

\section{Conclusion}

In this work, we measure experimentally the shape of the adhesive tape backing during steady peeling of a typical commercial PSA at controlled peeling angle $\theta$ and velocity $V$ with sufficient resolution to describe the debonding region. We model the tape backing by an elastica submitted to a constant force $F$ at the peeling end and to a uniform cohe- sive stress $\bar{\sigma}$ in the debonding region, where the adhesive material is stretched and forms fibrils. We make the reasonable assumption that this cohesive stress is oriented in the fibrils direction. Fitting the experimental profiles with such a model allows to extract the typical stress $\bar{\sigma}$ in the debonding region as well as the extension $a_{f}-a_{0}$ of the adhesive material at debonding, which are the effective rheological ingredients that determine the energy dissipated per peeled surface area $\Gamma=\bar{\sigma}\left(a_{f}-a_{0}\right)$ in this model. This fitting procedure also allows to compute the strain rate $\dot{\varepsilon}$ imposed to the adhesive fibrils and its average $\overline{\dot{\varepsilon}}$ in the debonding region. This model thus enables to use peeling as a sort of local rheological test giving access to the average stress, the average strain rate and the maximum extensibility of the adhesive in its complex fibrillar debonding region for a given set of control parameters $(\theta, V)$.

The model profiles fit properly the experimental ones down to the micrometric scale. We explain this agreement by the fact the model average stress $\bar{\sigma}$ is not too far from the actual stress distribution in the debonding region, since the stress-extension behavior of typical PSA is known from probe tack tests (which has a quite similar local loading configuration) to exhibit a large stress plateau, with similar typical stress values at corresponding strain rates (Lindner et al. 2004; Lindner et al. 2006). Moreover, except for very large peeling angles, the size of the cohesive zone and thus the fibrils extension is close to what can be observed by directly looking at the visible fibrils on the side of the tape. This is the first time to our knowledge that a curve displaying the typical stress and maximum strain in the adhesive debonding region during peeling versus the peeling angle and velocity (or versus the typical strain rate) is obtained and interpreted in terms of large strain rheology of the fibrillated adhesive material.

This use of peeling as a local rheological test of the complex debonding region should provide a useful tool to link the adhesive formulation to the desired user properties (i.e. adherence energy curves $\Gamma(V, \theta)$ ) through the intermediate parameters that are the material large strain rheological behaviour and fibril debonding conditions. While further studies on different formulations with well controlled large strain rheologies and interface properties should be conducted to completely examine this chain of influences, the parametric study in this paper already examines the influence of material's large-strain rheology on energy dissipation and thus resistance to peeling. Our study suggests that energy dissipation is linked to the peeling velocity $V$ mainly through the strain-rate dependency of the average stress within the adhesive, which seems to be insensitive to the peeling angle $\theta$, except maybe at the highest peeling angle, where other features such as plasticity in the tape backing are likely to occur. Conversely, the fibril elongation at debonding $a_{f}-a_{0}$ increases with $\theta$, but only slowly de- 
creases with $V$ in the explored velocity range. Finally and more speculatively, the angular dependency of $a_{f}$ (and thus the main angular dependency of $\Gamma$ ) could come from a change in the stress configuration at the fibrils extremities due to the changing direction $\varphi_{f}$ in which the fibrils are pulled, shifting the critical elongation at which the relevant debonding criterion (stress or energy density, see discussion in (Yarusso 1999)) is reached. In other words, our results suggest that longer fibril extensions can be reached before debonding and thus more energy can be dissipated when these fibrils are pulled with an angle $\varphi_{f}$ smaller than $90^{\circ}$ with respect to the substrate on which they are attached, provided the fibril attachment does not slide on the substrate (sliding being not observed in the experiments presented in this paper).

Finally and more generally, a consistent model to explain energy dissipation during peeling needs three ingredients: the equations of stress and displacement transmission between the operator and the zone where energy is dissipated; the effective rheological behavior of the adhesive in this zone; and a local debonding criterion. The modeling strategy presented in this paper accounts for the first point. We use a simplified version of the local adhesive rheology (a constant effective stress depending on the strain rate) to account for the second point, a simplification allowed by the large stress plateau exhibited by adhesives during probe tack tests. As for the third ingredient, our model gives access to observables that can shed some light on it, namely the elongation $a_{f}-a_{0}$ and orientation $\varphi_{f}$ of the fibril about to debond and the typical strain rate experienced by the fibrils.

Acknowledgements We thank B. Bresson and L. Olanier for their help in the conception of the experiments. We thank E. Barthel, M.J. Dalbe, S. Santucci, L. Vanel, and D. Yarusso for fruitful discussions. This work has been supported by the French ANR through Grant \#12BS09-014.

\section{References}

Amouroux et al. 2001. Amouroux N, Petit J, Léger L (2001) Role of interfacial resistance to shear stress on adhesive peel strength. Langmuir 17:6510-6517

Barquins and Ciccotti 1997. Barquins M, Ciccotti M (1997) On the kinetics of peeling of an adhesive tape under a constant imposed load. Int $\mathbf{J}$ Adhes Adhes 17:65-68

Chiche et al. 2005. Chiche A, Dollhofer J, Creton C (2005) Cavity growth in soft adhesives. Eur Phys J E 17:389401

Cortet et al. 2007. Cortet PP, Ciccotti M, Vanel L (2007) Imaging the stick-slip peeling of an adhesive tape under a constant load. J Stat Mech Theor Exp 2007:P03005
Cortet et al. 2013. Cortet PP, Dalbe MJ, Guerra C, Cohen C, Ciccotti M, Santucci S, Vanel L (2013) Intermittent stick-slip dynamics during the peeling of an adhesive tape from a roller. Phys Rev E 87:022601

Creton and Ciccotti 2016. Creton C, Ciccotti M (2016) Fracture and adhesion of soft materials : a review. Rep Prog Phys 79:046601

Creton et al. 2009. Creton C, Hu G, Deplace F, Morgret L, Shull KR (2009) Large-strain mechanical behavior of model block copolymer adhesives. Macromolecules 42:7605-7615

Dalbe et al. 2014a. Dalbe MJ, Santucci S, Cortet PP, Vanel L (2014a) Strong dynamical effects during stick-slip adhesive peeling. Soft Matter 10:132-138

Dalbe et al. 2014b. Dalbe MJ, Santucci S, Vanel L, Cortet PP (2014b) Peeling-angle dependence of the stick-slip instability during adhesive tape peeling. Soft Matter 10:9637-9643

Dalbe et al. 2015. Dalbe MJ, Cortet PP, Ciccotti M, Vanel L, Santucci S (2015) Multiscale stick-slip dynamics of adhesive tape peeling. Phys Rev Lett 115:128301

Deplace et al. 2009. Deplace, Creton C (2009) Fine tuning the adhesive properties of a soft nanostructured adhesive with rheological measurements. J Adhes 85:18-54

Derail et al. 1997. Derail C, Allal A, Marin G, Tordjeman P (1997) Relationship between viscoelastic and peeling properties of model adhesives. Part 1: Cohesive fracture. J Adhes 61:123-157

Derail et al. 1998. Derail C, Allal A, Marin G, Tordjeman PH (1998) Relationship between viscoelastic and peeling properties of model adhesives. Part 2: The interfacial fracture domains. J Adhes 68:203-228

Gent and Hamed 1977. Gent AN, Hamed GR (1977) Peel mechanics for an elastic-plastic adherend. J Appl Polym Sci 21:2817-2831

Gent and Petrich 1969. Gent AN, Petrich RP (1969) Adhesion of viscoelastic materials to rigid substrates. Proc Roy Soc A 310:433-448

ISO 8510-1. ISO 8510-1, Adhesives - Peel test for a flexible-bonded-to-rigid test specimen assembly - Part 1: $90^{\circ}$ degree peel (1990)

ISO 8510-2. ISO 8510-2, Adhesives - Peel test for a flexible-bonded-to-rigid test specimen assembly - Part 2: $180^{\circ}$ degree peel (2006)

Kaelble 1959. Kaelble DH (1959) Theory and analysis of peel adhesion: Mechanisms and mechanics. Trans Soc Rheol 3:161-180

Kaelble 1960. Kaelble DH (1960) Theory and analysis of peel adhesion: Bond stresses and distributions. Trans Soc Rheol 4:45-73

Kaelble 1965. Kaelble DH (1965) Peel adhesion: Microfracture mechanics of interfacial unbonding of polymers. Trans Soc Rheol 9:135-163 
Kendall 1975. Kendall K (1975) Thin-film peeling-the elastic term. J Phys D Appl Phys 8:1449-1452

Lakrout et al. 1999. Lakrout H, Sergot P, Creton C (1999) Direct observation of cavitation and fibrillation in a probe tack experiment on model acrylic PressureSensitive-Adhesives. J Adhes 69:307-359

Lindner et al. 2004. Lindner A, Maevis T, Brummer R, Lühmann B, Creton C (2004) Subcritical failure of soft acrylic adhesives under tensile stress. Langmuir 20:9156-9169

Lindner et al. 2006. Lindner A, Lestriez B, Mariot S, Creton C, Meavis T, Lühmann B, Brummer R (2006) Adhesive and rheological properties of lightly crosslinked model acrylic networks. J Adhes 82:267-310

Love 1944. Love AEH (1944) A treatise on the mathematical theory of elasticity. Dover Publications, New York

Niesiolowski and Aubrey 1981. Niesiolowski F, Aubrey DW (1981) Stress distribution during peeling of adhesive tapes. J Adhes 13:87-98

Villey et al. 2015. Villey R, Creton C, Cortet PP, Dalbe MJ, Jet T, Saintyves B, Santucci S, Vanel L, Yarusso DJ, Ciccotti M (2015) Rate-dependent elastic hysteresis during the peeling of pressure sensitive adhesives. Soft Matter 11:3480-3491

Yarusso 1999. Yarusso DJ (1999) Quantifying the relationship between peel and rheology for Pressure Sensitive Adhesives. J Adhes 70:299-320 